\title{
Efficacy and Safety of Endoscopic Submucosal Dissection for Superficial Gastric Neoplasms: A Latin American Cohort Study
}

\author{
Fernando Palacios-Salas ${ }^{1}$, Harold Benites-Goñi ${ }^{2,3}$, Luis Marin-Calderón ${ }^{3}$, Paulo Bardalez-Cruz ${ }^{3}$, Jorge Vásquez-Quiroga ${ }^{3}$, Edgar \\ Alva-Alva ${ }^{3}$, Bryan Medina-Morales ${ }^{3}$, and Jairo Asencios-Cusihuallpa ${ }^{3}$ \\ ${ }^{1}$ Clínica Delgado y Oncosalud - AUNA, Lima, ${ }^{2}$ Vicerrectorado de Investigación, Universidad San Ignacio de Loyola, Lima, ${ }^{3} \mathrm{Hospital}$ \\ Nacional Edgardo Rebagliati Martins, Lima, Perú
}

Background/Aims: Endoscopic submucosal dissection (ESD) is the preferred technique for treating early gastric cancer (EGC). However, very few studies have been conducted in South America. This study aimed to assess the efficacy and safety of ESD for EGC. Methods: We analyzed data from a prospective cohort from 2013 to 2020 . A total of 152 superficial gastric neoplasms that fulfilled the absolute or expanded criteria for ESD were included. Outcomes were en bloc, R0, and curative resection rates, incidence of adverse events, and length of procedure.

Results: The age of the enrolled patients was $68.4 \pm 11.3$ years. The number of included patients based on the absolute and expanded indications was 150 and 2, respectively. En bloc, R0, and curative resections were achieved in 98.0\%, 96.1\%, and 89.5\% of the cases, respectively. Bleeding and perforation were reported in $5.9 \%$ and $6.6 \%$ of the cases, respectively. Histopathological examination revealed low-grade dysplasia, high-grade dysplasia, well-differentiated adenocarcinoma, and poorly differentiated adenocarcinoma in $13,20,117$, and 2 cases, respectively.

Conclusions: Our study shows that ESD performed by properly trained endoscopists in reference centers is safe and effective, with comparable therapeutic outcomes to those reported in the Eastern series. Clin Endosc 2022;55:248-255

Key Words: Adverse effects; Endoscopic submucosal dissection; Gastric cancer; Treatment outcome

\section{INTRODUCTION}

Gastric cancer is a frequent malignant tumor and the third leading malignant cause of death worldwide. ${ }^{1}$ South America has a high incidence of gastric cancer, with 49,547 new cases annually. In Peru, the estimated incidence is 6,300 new cases annually (male-to-female ratio, 1.2), most of which are diagnosed at an advanced stage. ${ }^{1}$ Although mortality rates have decreased over the past years, gastric cancer is the leading malignant cause of death locally. ${ }^{1,2}$

Received: June 17,2021 Revised: July 5, 2021

Accepted: July 22, 2021

Correspondence: Harold Benites-Goñi

Calle Mozart 143, San Borja 15037, Lima, Perú

Tel: +51-998-822-811 , Fax: +51-685-5365, E-mail: hbenites@usil.edu.pe

ORCID: https://orcid.org/0000-0003-2083-1840

(cc) This is an Open Access article distributed under the terms of the Creative Commons Attribution Non-Commercial License (http://creativecommons.org/ licenses/by-nc/3.0) which permits unrestricted non-commercial use, distribution, and reproduction in any medium, provided the original work is properly cited.
Early diagnosis of gastric cancer increases the chances of curative treatment. In Japan and other Asian countries, the treatment of choice for early gastric cancer (EGC) is endoscopic resection, showing excellent short- and long-term results. ${ }^{3-7}$ Endoscopic submucosal dissection (ESD) is the preferred resection technique, as it allows faster recovery and better quality of life than surgery. ${ }^{8}$

To use ESD as a standard treatment for EGC in Western countries, similarly good results to those in Eastern studies need to be achieved. Only a few studies have been published in Western countries, given the low incidence of gastric cancer and the technical complexity of ESD. ${ }^{9-13}$ Therefore, this study aimed to assess the therapeutic outcomes and safety of ESD for EGC in two endoscopy centers in Peru.

\section{MATERIALS AND METHODS}

Data were collected and analyzed from a prospective observational cohort of all patients referred for ESD of biopsy-con- 
firmed EGC or adenomatous dysplasia at two medical centers in Peru. All lesions met the indications proposed by the latest Japanese Gastric Cancer Treatment Guidelines (JGCTG) for endoscopic resection of EGC. ${ }^{14}$ The precursors of EGC were classified as low-grade dysplasia (LGD) and high-grade dysplasia (HGD). We excluded patients with lesions clinically predicting massive submucosal invasion, or with evidence of lymph node or distant metastases.

\section{Endoscopic characteristics of the lesions}

The Japanese Classification of Gastric Carcinoma was used to determine the location and macroscopic type of the lesion. ${ }^{15}$ The stomach was divided into upper, middle, and lower third regions. The macroscopic type was classified as elevated (I and IIa) or depressed/flat (IIb and IIc) and was described using the Paris classification. ${ }^{16}$ The lesions were measured in centimeters. The presence of ulcers was defined based on the endoscopic or pathological findings. The clinical prediction of invasion depth was made by conventional endoscopy according to the criteria proposed by Palacios et al. ${ }^{17}$ The indications for ESD were categorized as absolute, expanded, or relative.

\section{ESD}

ESDs were performed by four endoscopists (FPS, LMC, $\mathrm{PBC}, \mathrm{JVQ}$ ) with previous skills in therapeutic endoscopy and specialized training on ESD, locally and abroad, with renowned Japanese professors. The senior endoscopist (FPS) mentored the training and ESDs for all team members. Sedoanalgesia and general anesthesia were eventually performed.

The equipments used in most cases were Fujinon, EG530WR, EG-590WR, EG-590ZW, and EG-600ZW endoscopes (Fujinon Co., Ltd, Tokyo, Japan); Olympus GIF-HQ190 endoscopes (Olympus Optical Co., Ltd, Tokyo, Japan); ERBE VIO200D electrosurgical source (ERBE elektromedizin GmBH, Tübingen, Germany) and a carbon dioxide insufflation system. The endoscopic knives used were mostly DualKnife (KD-650L; Olympus, Tokyo, Japan), sometimes, HybridKnife (ERBEJET 2; ERBE elektromedizin GmBH, Tübingen, Germany), and rarely, the ITknife2 (KD-611L; Olympus, Tokyo, Japan).

The lesions were characterized and delimited with Fuji intelligent color enhancement, narrow-band imaging, magnification, and sequential chromoendoscopy with $1.5 \%$ acetic acid and $0.3 \%$ indigo carmine. The lesions were marked 3-5 $\mathrm{mm}$ around them and separated 3-5 $\mathrm{mm}$ from each other using argon plasma (Forced APC $25 \mathrm{~W}$; ERBE elektromedizin $\mathrm{GmBH}$, Tübingen, Germany) or the tip of the DualKnife (KD650L; Olympus, Tokyo, Japan) (Soft COAG, E5 100 W).

A transparent cap placed on the endoscope tip (straight or conical) with a 3-4-mm protusion was used in all the procedures. The lesions were elevated by sequential submucosal injections of $10 \%$ mannitol (previously diluted with $0.9 \%$ sodium chloride) with 1:250,000 diluted adrenaline, and slightly stained with methylene blue. A partial incision of 3-5 $\mathrm{mm}$ around the margin of each lesion was made, followed by submucosal dissection until approximately $60 \%-70 \%$ of the lesion. The electrosurgical unit settings were mostly ENDO CUT I E3-3-3 and Swift COAG E4 $40 \mathrm{~W}$ for cutting and dissection, respectively. Visible small vessels during dissection were thermocoagulated with the same endoscopic knives, whereas larger vessels were thermocoagulated with the coagrasper hemostatic forceps (FD-410 LR; Olympus, Tokyo, Japan) or hot biopsy forceps (Soft COAG, E5 $80 \mathrm{~W}$ was used in both cases). In cases of large and pulsating vessels, hemoclips were applied. Procedure time was defined as the time from the first incision to completion of the dissection. Figures 1 and 2 show the sequential ESD images.

\section{Histologic examination}

The samples were fixed in $10 \%$ formalin. The maximum diameter of each lesion and specimen was measured. The specimens were then sectioned at 2-mm intervals. The histological type of each lesion was determined based on the Vienna and World Health Organization criteria. ${ }^{18,19}$ Horizontal and vertical margins, depth of invasion, lymphovascular invasion, and presence of ulcerations were evaluated. Depth of invasion was determined as mucosal (M or pT1a), superficial submucosal $(<500 \mu \mathrm{m}, \mathrm{SM} 1$ or pT1b1), or deep submucosal $(\geq 500 \mu \mathrm{m}$, SM2 or pT1b2).

\section{Adverse events and post-procedure care}

Adverse events were defined according to the consensus of the American Society for Gastrointestinal Endoscopy for endoscopic adverse effects. ${ }^{20}$ Significant immediate bleeding ( $>2$ $\mathrm{g} / \mathrm{dl}$ drop in hemoglobin, or requirement of transfusion) was considered an adverse event. Delayed bleeding was considered as occurring within 30 days after ESD, with the same clinical characteristics as immediate bleeding and/or the need for an intervention.

Perforation was defined as a full-thickness defect during ESD or the presence of air or luminal content outside the gastrointestinal tract. Immediate perforation was recognized and treated with hemoclips. Surgery was indicated if hemoclip closure was unsuccessful. Delayed perforation was considered as occurring within the first 30 days after ESD, and the indicated treatment was surgical.

\section{Definitions}

En bloc resection: Resection of neoplastic lesion in one single piece. 


\section{Ce cuncan enooscopy}
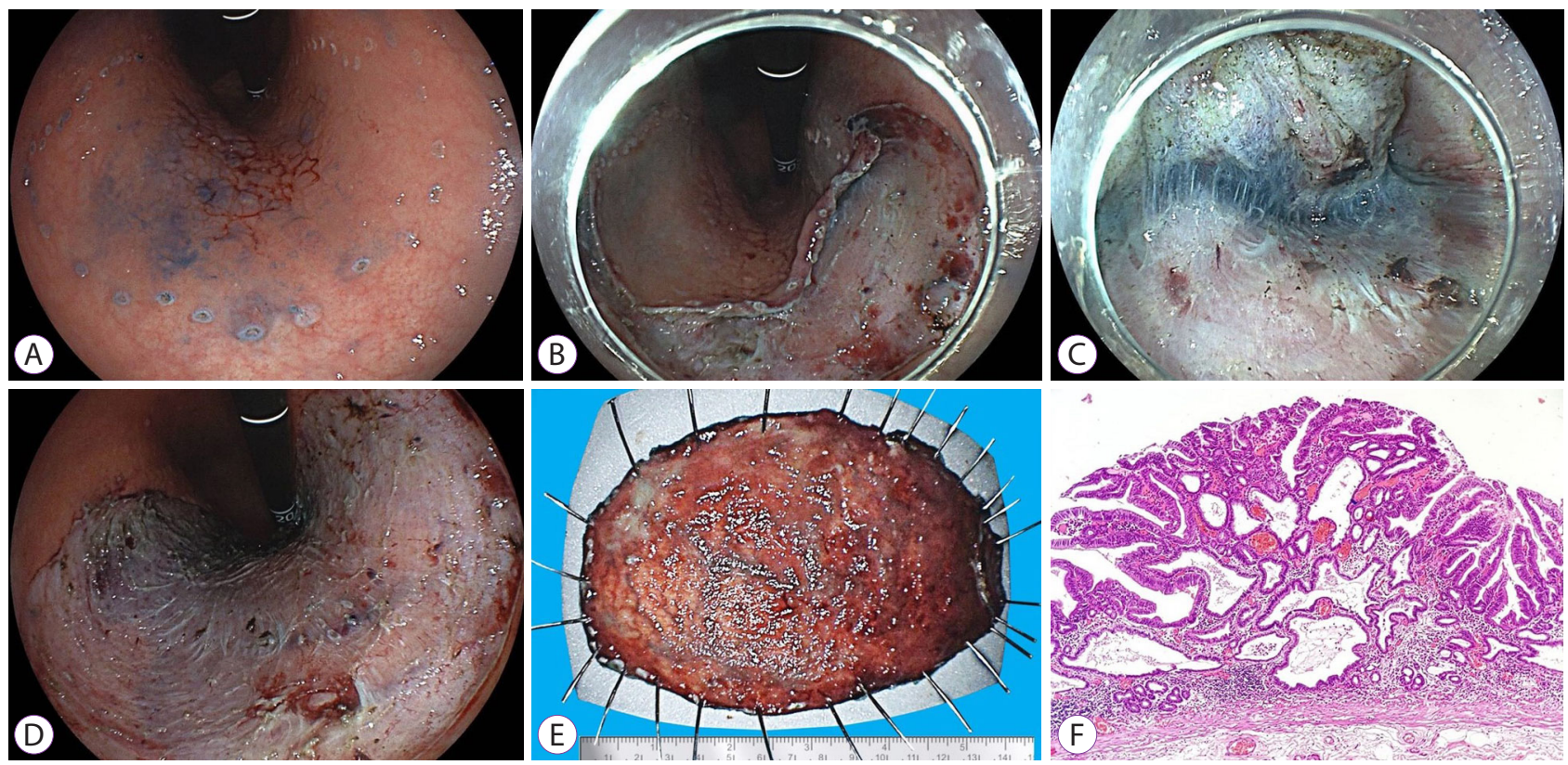

Fig. 1. Endoscopic submucosal dissection of an early gastric cancer (0-llb+lla) on the gastric body. (A) Marking outside the lesion. (B) Peripheral partial incision. (C) Submucosal dissection. (D) Post-resection ulcer. (E) Fixation of the tissue specimen. (F) Low-power view of the resected specimen shows differentiated adenocarcinoma (hematoxylin \& eosin stain, $\times 40$ ).
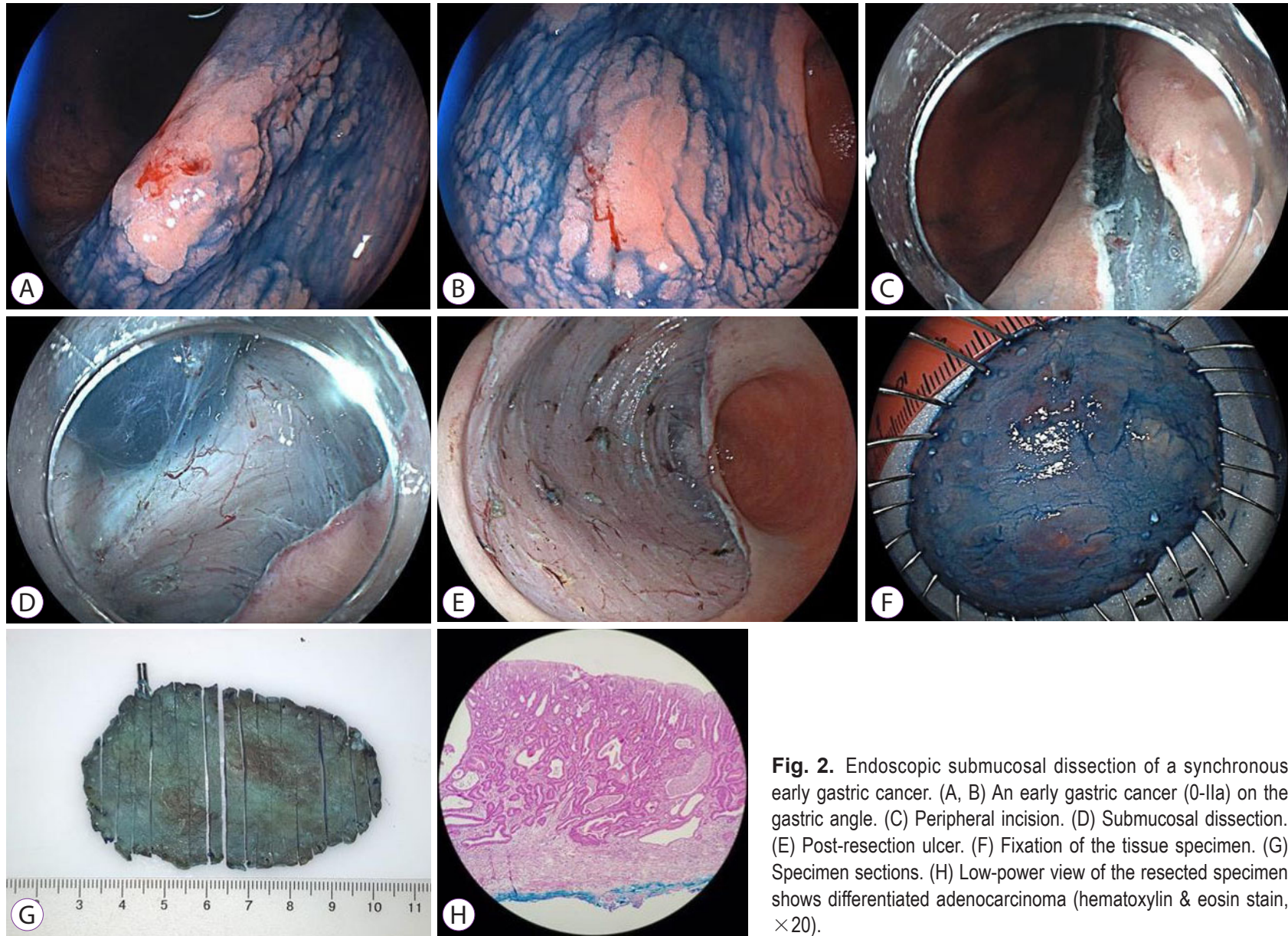

Fig. 2. Endoscopic submucosal dissection of a synchronous early gastric cancer. (A, B) An early gastric cancer (0-lla) on the gastric angle. (C) Peripheral incision. (D) Submucosal dissection. (E) Post-resection ulcer. (F) Fixation of the tissue specimen. (G) Specimen sections. (H) Low-power view of the resected specimen shows differentiated adenocarcinoma (hematoxylin \& eosin stain, $\times 20$ ). 
Complete resection (R0): En bloc resected lesion with histologic confirmation of horizontal and vertical margins free of neoplasia (HM0 and VM0). If there were any compromised margins due to neoplasia (HM1 and/or VM1), the resection was considered "not complete" (R1). Piecemeal resection of up to three pieces can be $\mathrm{R} 0$ if reconstruction is possible; otherwise, it does not allow the microscopic evaluation of the horizontal margins; therefore, the resection would be considered R1. If a neoplastic lesion could not be removed, it was considered as "failed resection".

\section{Curability criteria}

In cases of categories IV and V, according to the Vienna classification (HGD and adenocarcinoma, respectively), the curability criteria followed the definitions proposed by JGCTG. ${ }^{14}$

- Endoscopic curability A (eCuraA): En bloc resection without ulcerative findings (UL0), any tumor size, histologically differentiated type-dominant, pT1a, HM0, VM0, and no lymphovascular invasion (Ly-V0). If ulcerative findings are present (UL1), the resection is still classified as eCuraA when the tumor size is $\leq 3 \mathrm{~cm}$ and the other conditions are met. However, if the undifferentiated component of the lesion $>2 \mathrm{~cm}$ in length, the endoscopic curability is classified as C-2 (eCuraC-2).

- Endoscopic curability B (eCuraB): En bloc resection, HM0, VM0, Ly-V0, and fulfilling one of the following conditions: (a) pT1a cancer, histologically undifferentiated type-dominant, UL0, tumor size $\leq 2 \mathrm{~cm}$; (b) pT1b cancer, histologically differentiated type-dominant, pT1b1 (SM1) $(<500 \mu \mathrm{m}$ from the muscularis mucosa), tumor size $\leq 3 \mathrm{~cm}$. However, if the undifferentiated component is included in the portion with submucosal invasion, the endoscopic curability is classified as C-2 (eCuraC-2).

- Endoscopic curability $\mathrm{C}$ (eCuraC): The resection is classified as endoscopic curability $\mathrm{C}$ (eCuraC) when it does not fulfill the conditions described above to be classified as either eCuraA or eCuraB. An eCuraC-1 resection is referred to cases only with HM1, while an eCuraC-2 resection was used for cases with other non-curative conditions, such as VM1, SM2 invasion, or Ly-V1.

Categories eCuraA and eCuraB were considered curative resections. Category eCuraC was considered a noncurative resection. In the case of category III of the Vienna classification (LGD), curative resection was defined if resection met the R0 criteria.

\section{Statistical analysis}

Statistical analysis was performed using SPSS (version 23;
IBM Corp., Armonk, NY, USA). Frequencies and percentages were calculated for categorical variables, and means and standard deviations or medians and interquartile ranges (IQR) were calculated for continuous variables. Qualitative and quantitative variables were evaluated using the Fisher exact test and Student's $t$-test or Mann-Whitney $U$ test, respectively. The significance of differences in the size and duration of the procedure by curative resection was evaluated by the Kruskal-Wallis test. Statistical significance was set at $p<0.05$.

\section{Ethical considerations}

The protocol was approved by the local ethics committee (832-2021-030). Informed consent was waived because of the retrospective study design and because the analysis used anonymous clinical data. The study protocol conformed to the ethical guidelines of the 1975 Declaration of Helsinki.

\section{RESULTS}

Between January 2013 and July 2020, 137 patients from two reference medical centers with 152 superficial gastric neoplasms were included.

\section{Patient and lesion characteristics}

Patient characteristics and resected lesions are shown in Table 1. The mean age was 68.4 years (range 28-94 years). The female-to-male ratio was 1.4. The median lesion size was $1.8 \mathrm{~cm}$ (IQR, $1.5-2.6 \mathrm{~cm}$ ), and the size of the largest resected lesion was $12 \mathrm{~cm}$. The indication for ESD was absolute in 150 cases, according to the revised JGCTG criteria (98.7\%).

\section{ESD procedures}

The characteristics of the procedures are described in Table 2. Immediate perforation occurred in nine cases (5.9\%), of which eight were successfully treated using through-the-scope clips, and only one required surgery because of the difficult location for clipping (pyloroduodenal extension of antral lesion). Delayed perforation occurred in one case $(0.7 \%)$ after difficult ESD at the gastrojejunal anastomosis, which was treated surgically. All bleeding cases were managed endoscopically. No deaths were associated with ESD.

\section{Histological analysis}

Complete resection was achieved in 146 out of 152 cases (96.1\%). The histopathology of the lesions is described in Table 3 . 
Table 1. Patient and Lesion Characteristics

\begin{tabular}{|c|c|}
\hline Clinical characteristics & $n(\%)$ \\
\hline \multicolumn{2}{|l|}{ Sex } \\
\hline Female & $80(52.6)$ \\
\hline Mean age, years (SD) & $68.4( \pm 11.3)$ \\
\hline \multicolumn{2}{|l|}{ Localization } \\
\hline Upper third & $10(6.6)$ \\
\hline Middle third & $50(32.9)$ \\
\hline Lower third & $92(60.5)$ \\
\hline \multicolumn{2}{|l|}{ Paris classification type } \\
\hline $0-\mathrm{I}$ & $18(11.8)$ \\
\hline 0-IIa & $45(29.6)$ \\
\hline 0 -IIb & $5(3.3)$ \\
\hline 0-IIc & $41(27.0)$ \\
\hline Mixed type & $43(28.3)$ \\
\hline \multicolumn{2}{|l|}{ Macroscopic type } \\
\hline Elevated type & $70(46.1)$ \\
\hline Depressed/flat type & $82(53.9)$ \\
\hline \multicolumn{2}{|c|}{ Lesion size (measured after resection) } \\
\hline$\leq 2 \mathrm{~cm}$ & $88(57.9)$ \\
\hline $2-3 \mathrm{~cm}$ & $44(28.9)$ \\
\hline$>3 \mathrm{~cm}$ & $20(13.2)$ \\
\hline \multicolumn{2}{|l|}{ Ulceration } \\
\hline Present & $8(5.3)$ \\
\hline \multicolumn{2}{|l|}{ Indication for ESD } \\
\hline Absolute & $150(98.7)$ \\
\hline Expanded & $2(1.3)$ \\
\hline
\end{tabular}

ESD, endoscopic submucosal dissection; SD, standard deviations.
Table 3. Histological Analysis

\begin{tabular}{lc}
\hline Histological analysis & $\boldsymbol{n}(\mathbf{\%})$ \\
\hline Histology of the lesion & \\
Low-grade dysplasia & $13(8.6)$ \\
High-grade dysplasia & $20(13.2)$ \\
Differentiated adenocarcinoma & $117(77.0)$ \\
Undifferentiated adenocarcinoma & $2(1.3)$ \\
Invasion & \\
M & $139(91.4)$ \\
SM1 & $2(1.3)$ \\
SM2 & $11(7.2)$ \\
Horizontal margins & \\
HM0 & $146(96.1)$ \\
HM1 & $4(2.6)$ \\
HMx & $2(1.3)$ \\
Vertical margins & \\
VM0 & $151(99.3)$ \\
VM1 & $0(0)$ \\
VMx & $1(0.7)$ \\
Lymphovascular invasion & $1(0.7)$ \\
Complete resection & $146(96.1)$ \\
\hline
\end{tabular}

HM0, negative horizontal margin; HM1, positive horizontal margin; $\mathrm{HMx}$, unclear horizontal margin; $\mathrm{M}$, lesion confined to mucosa; SM1, cancer with depth of invasion from the muscularis mucosa $<500 \mu \mathrm{m}$; SM2, cancer with depth of invasion from the muscularis mucosa $\geq 500 \mu \mathrm{m}$; VM0, negative vertical margin; VM1, positive vertical margin; VMX, unclear vertical margin.

\section{Evaluation of curability}

Thirteen resected lesions by LGD were considered curative. Of 139 lesions with HGD and adenocarcinoma, 120 (86.3\%), $3(2.2 \%), 4(2.9 \%)$, and 12 (8.6\%) were categorized as eCuraA, eCuraB, eCuraC-1, and eCuraC-2, respectively, with two eCuraC-2 lesions in the same patient. Fig. 3 summarizes the indications for ESD and the main outcomes.

In considering lesions with LGD and lesions within categories eCuraA and eCuraB, the global curative resection rate was $89.5 \%$ (136/152). Regarding lesions within categories eCuraC-1 and eCuraC-2, the grouped non-curative resection rate was $10.5 \%$ (16/152). Of 15 patients with non-curative resections, four underwent surgery, all of whom had lesions with $\mathrm{R} 0$ and eCuraC-2 resections. Postoperative residual neoplastic tissues were found in the resection area in two cases, and none had lymph node metastases. 


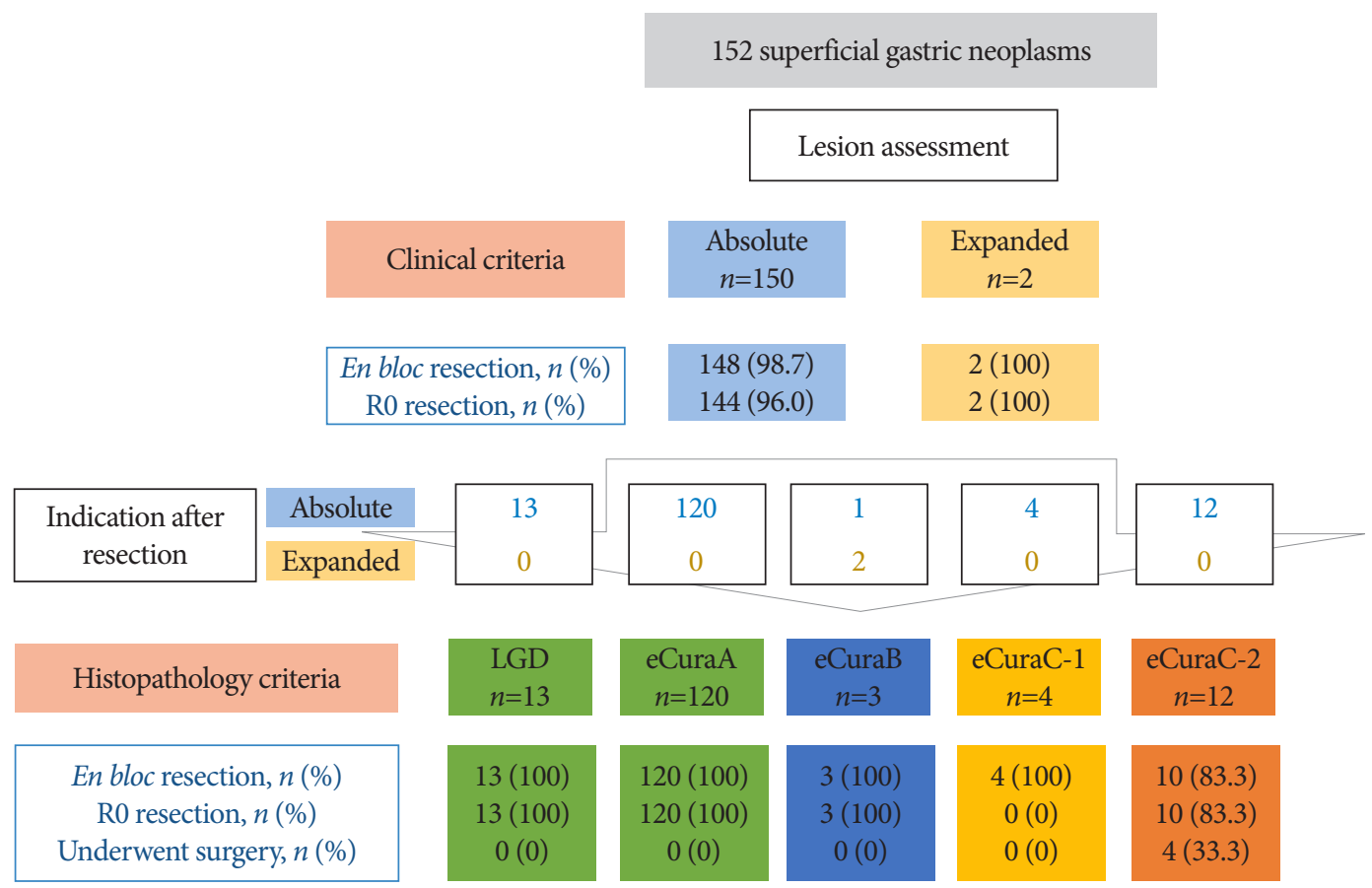

Fig. 3. Indications for endoscopic submucosal dissection, main outcomes and curability criteria. LGD, low-grade dysplasia.

\section{DISCUSSION}

The recent development of ESD for treating EGC has shown excellent short- and long-term results. ${ }^{14}$ ESD has been developed in Japan and has advantages over endoscopic mucosal resection in treating EGC, in terms of the probability of en bloc resection without any size limitation, which results in an improved histological assessment, higher complete and curative resection rates, and lower local recurrence. ${ }^{21,22}$

To date, the majority of studies on this topic have been conducted in Eastern countries, reflecting their great experience in ESD, becoming their preferred approach in the treatment of EGC. In contrast, a lower incidence of gastric cancer and a higher proportion of lesions found in advanced stages in Western countries have delayed the learning and implementation of ESD. To consider this technique as the standard treatment for EGC in non-Eastern countries, the same good results achieved in Asian studies must be achieved.

In our study, we achieved high en bloc, R0, and curative resection rates at $98.0 \%, 96.1 \%$, and $89.5 \%$, respectively, which are similar to those in Japanese and Korean studies (92\%-99.4\%, 90.1\%-97.3\%, and 73.6\%-94.7\%, respectively). ${ }^{22-26}$ A systematic review including six Latin American studies, revealed en bloc, R0, and curative overall resection rates of $96 \%, 84 \%$, and $72 \%$, respectively. ${ }^{27}$ To our knowledge, the largest Western series of ESD for EGC has recently been published by Ngamruengphong et al., ${ }^{28}$ who reported en bloc, $\mathrm{R} 0$, and curative resection rates of $92.2 \%, 91.8 \%$, and $58.7 \%$, from 347 lesions resected in several hospitals in the United States. An important Latin American study by Arantes et al. ${ }^{13}$ reported achieving en bloc and $\mathrm{R} 0$ resection rates of $>90 \%$ and a curative resection rate of $76 \%$ in 35 adenomas and 42 EGCs resected by ESD. The curative resection rates in both studies are lower than that in our study.

One reason for our high-quality results, specifically our high curative resection rate, was the accuracy in diagnosing the invasion depth of the lesions. Previously, Palacios et al. ${ }^{17}$ proposed five endoscopic features that could predict massive submucosal invasion in EGC as follows: margin and central elevation with submucosal appearance, irregular surface, enlarged folds, size $>30 \mathrm{~mm}$ in protruding lesions, and lesion rigidity. In a validation study, the presence of two or more of these characteristics predicted massive submucosal invasion with very high accuracy and positive predictive value; therefore, these lesions should not be treated by ESD. Another important reason is the systematic training on ESD described previously.

The updated JGCTG included new indications for endoscopic resection and definitions of curability, ${ }^{14}$ based on the results of the JCOG0607 study, which showed a similar 5-year overall survival rate to that of the general population, in pa- 
tients after ESD of EGC that satisfied the previous expanded criteria of differentiated-type mucosal gastric cancer and UL0 tumors $>2 \mathrm{~cm}$ in size or UL1 tumors $\leq 3 \mathrm{~cm}$ in size. ${ }^{29}$ These categories are now considered absolute indications for ESD, and after satisfying curative criteria are categorized as eCuraA. In our study, almost all lesions had absolute indications for ESD, and most were categorized as eCuraA after resection. Only two lesions with undifferentiated-type mucosal gastric cancer, UL0, and $\leq 2 \mathrm{~cm}$ in size had expanded indications for ESD, and these two lesions and one differentiated-type gastric cancer with tumor size $\leq 3 \mathrm{~cm}$ and SM1 invasion, fulfilled the new expanded curative criteria and were categorized as eCur$\mathrm{aB}$ after resection. Resections were considered noncurative in 16 cases, of which four with local recurrence risk because of HM1 were categorized as eCuraC-1, and 12 with risk of lymph node metastases because of SM2 invasion, lymphovascular invasion, or VM1, were categorized as eCuraC-2. Ten of the 12 eCuraC-2 resections had $\mathrm{R} 0$ resections.

The median size of the resected lesions and the specimens were $1.8 \mathrm{~cm}$ and $3.5 \mathrm{~cm}$, respectively. The largest successfully treated lesion was $12 \mathrm{~cm}$ in size, located in the lesser curvature of the middle and upper thirds of the stomach, representing the consolidation of our ESD learning curve. The median operating time was $53.5 \mathrm{~min}$, which was similar to that reported in Eastern studies and lower than that reported in Western countries (61-135 min). ${ }^{9-13,23}$

Regarding adverse events, perforation was immediate in nine cases (5.9\%) and delayed in one case (0.7\%). Most perforations were treated successfully by placing hemoclips, but one case of immediate perforation (occurring in a lesion extending through the pylorus to the duodenal bulb) and delayed perforation required surgery. Our perforation rate was slightly higher than that reported by Oda in a review of 28 Japanese and Korean studies, including $>300$ gastric ESD each, which ranged from $1.2 \%-5.2 \%$, and also higher than the values reported by Zullo et al. in a meta-analysis of Western studies with an overall rate of 3.4\% (95\% confidence interval [CI], 2.5\%-4.6\%). ${ }^{27,30}$ However, as Zullo et al. ${ }^{27}$ pointed out, the perforation rate was higher in studies with few patients, even reaching $22 \%$. The perforation rate is closely related to the learning curve. Seven of the nine cases of immediate perforation occurred in the first 50 ESDs. In addition, we initially worked with standard gastroscopes, without high definition or accessory water irrigation channels, and most procedures were performed with sedoanalgesia, which is a non-general anesthesia, as reported by most Western series, increasing technical difficulty. Delayed perforation was reported in $0.5 \%$ of cases in a large Japanese study, similar to ours. ${ }^{31}$

Delayed bleeding is another important adverse event associated with gastric ESD. The incidence was 3.9\%, and all cases

were treated using endoscopy. In Eastern studies, delayed bleeding has been reported to range from $0 \%$ to $15.6 \% .^{30}$ In most Eastern centers, a second-look endoscopy is performed the day after ESD, which is not usually performed if there is no evidence of bleeding. A meta-analysis of Western studies revealed an overall delayed bleeding rate of 5.8\% (95\% CI, $4.6 \%-7.3 \%)^{27}$

The strength of this study is that it has one of the largest cohorts of patients with EGC and premalignant gastric lesions treated by ESD in non-Eastern countries with the largest proportion in Latin America. The limitations of this study are the relatively small number of patients compared to those in Eastern studies, its retrospective design, and unavailable shortterm data. Furthermore, the possibility of Helicobacter pylori infection was not considered in our study. This variable should be considered in subsequent studies because of its high local incidence (approximately 50\%) and relationship with gastric cancer development, which could increase the risk of recurrence. $^{32}$

In conclusion, our study shows that ESD performed by properly trained endoscopists in local reference centers is safe and effective, with comparable en bloc, complete, and curative resection rates and similar adverse events to those reported by Eastern studies. Thus, ESD should be established as a standard treatment for EGC in our country, with almost no risk of lymphnode metastasis. Further data are needed to define the long-term outcomes.

Conflicts of Interest

The authors have no potential conflicts of interest

Funding

None.

Author Contributions

Conceptualization: Fernando Palacios-Salas, Harold Benites-Goñi Data curation: FPS, HBG

Formal analysis: $\mathrm{HBG}$

Investigation: FPS, HBG, Luis Marin-Calderón, Paulo Bardalez-Cruz, Jorge Vasquez-Quiroga, Edgar Alva-Alva, Bryan Medina-Morales, Jairo Asencios-Cusihuallpa

Methodology: FPS, HBG

Supervision: FPS, HBG

Validation: FPS, HBG

Writing-original draft: FPS, HBG

Writing-review \& editing: FPS, HBG

ORCID

Fernando Palacios-Salas Harold Benites-Goñi Luis Marin-Calderón Paulo Bardalez-Cruz Jorge Vásquez-Quiroga Edgar Alva-Alva https://orcid.org/0000-0003-2229-2238 https://orcid.org/0000-0003-2083-1840 https://orcid.org/0000-0002-3407-1902 https://orcid.org/0000-0001-6910-7090 https://orcid.org/0000-0003-1451-4590 https://orcid.org/0000-0003-1259-0522 
Bryan Medina-Morales

Jairo Asencios-Cusihuallpa

https://orcid.org/0000-0001-7158-761X https://orcid.org/0000-0002-6182-0518

\section{REFERENCES}

1. Sung H, Ferlay J, Siegel RL, et al. Global cancer statistics 2020: GLOBOCAN estimates of incidence and mortality worldwide for 36 cancers in 185 countries. CA Cancer J Clin 2021;71:209-249.

2. Zafra-Tanaka JH, Tenorio-Mucha J, Villarreal-Zegarra D, Carrillo-Larco R, Bernabe-Ortiz A. Cancer-related mortality in Peru: trends from 2003 to 2016. PLoS One 2020;15:e228867.

3. Suzuki H, Oda I, Abe S, et al. High rate of 5-year survival among patients with early gastric cancer undergoing curative endoscopic submucosal dissection. Gastric Cancer 2016;19:198-205.

4. Kim SG, Park CM, Lee NR, et al. Long-term clinical outcomes of endoscopic submucosal dissection in patients with early gastric cancer: a prospective multicenter cohort study. Gut Liver 2018;12:402-410.

5. Kosaka T, Endo M, Toya Y, et al. Long-term outcomes of endoscopic submucosal dissection for early gastric cancer: a single-center retrospective study. Dig Endosc 2014;26:183-191.

6. Choi MK, Kim GH, Park DY, et al. Long-term outcomes of endoscopic submucosal dissection for early gastric cancer: a single-center experience. Surg Endosc 2013;27:4250-4258.

7. Tanabe S, Ishido K, Matsumoto T, et al. Long-term outcomes of endoscopic submucosal dissection for early gastric cancer: a multicenter collaborative study. Gastric Cancer 2017;20:45-52.

8. Liu Q, Ding L, Qiu X, Meng F. Updated evaluation of endoscopic submucosal dissection versus surgery for early gastric cancer: a systematic review and meta-analysis. Int J Surg 2020;73:28-41.

9. Chaves DM, Maluf Filho F, de Moura EGH, et al. Endoscopic submucosal dissection for the treatment of early esophageal and gastric cancer-initial experience of a western center. Clinics (Sao Paulo) 2010;65:377-382.

10. Donoso D A, Sharp A, Parra-Blanco A, et al. Endoscopic submucosal dissection in early gastric cancer: experience in 16 patients. Rev Med Chil 2015;143:1277-1285.

11. Emura F, Mejía J, Donneys A, et al. Therapeutic outcomes of endoscopic submucosal dissection of differentiated early gastric cancer in a Western endoscopy setting (with video). Gastrointest Endosc 2015;82:804-811.

12. Chirinos Vega JA, Vargas G, Alcántara C, Zapata J. Endoscopic submucosal dissection as treatment for early gastric cancer: experience at two centers in Lima, Peru. Rev Gastroenterol Mex (Engl Ed) 2018;83:393399.

13. Arantes V, Aliaga Ramos J, Pedrosa MS. Endoscopic submucosal dissection for superficial gastric neoplasias in two referral hospitals in Brazil: can the Japanese and South Korean results be equaled? Rev Gastroenterol Mex 2021;86:244-252.

14. Japanese Gastric Cancer Association. Japanese gastric cancer treatment guidelines 2018 (5th edition). Gastric Cancer 2021;24:1-21.

15. Japanese Gastric Cancer Association. Japanese classification of gastric carcinoma: 3rd English edition. Gastric Cancer 2011;14:101-112.

16. Participants in the Paris Workshop. The Paris endoscopic classification of superficial neoplastic lesions: esophagus, stomach, and colon: November 30 to December 1, 2002. Gastrointest Endosc 2003;58:S3-S43.

17. Palacios Salas F, Liza Baca E. Endoscopic prediction of tumor invasion depth in early gastric neoplasia: a prospective study in Peru. Rev Gastroenterol Peru 2017;37:120-128.

18. Hu B, El Hajj N, Sittler S, Lammert N, Barnes R, Meloni-Ehrig A. Gastric cancer: classification, histology and application of molecular pathology. J Gastrointest Oncol 2012;3:251-261.

19. Schlemper RJ, Riddell RH, Kato Y, et al. The Vienna classification of gastrointestinal epithelial neoplasia. Gut 2000;47:251-255.

20. Cotton PB, Eisen GM, Aabakken L, et al. A lexicon for endoscopic adverse events: report of an ASGE workshop. Gastrointest Endosc 2010;71:446-454.

21. Gotoda T, Kondo H, Ono H, et al. A new endoscopic mucosal resection procedure using an insulation-tipped electrosurgical knife for rectal flat lesions: report of two cases. Gastrointest Endosc 1999;50:560-563.

22. Oda I, Saito D, Tada M, et al. A multicenter retrospective study of endoscopic resection for early gastric cancer. Gastric Cancer 2006;9:262-270.

23. Goto A, Nishikawa J, Okamoto T, et al. Outcomes of endoscopic submucosal dissection for early gastric cancer and factors associated with incomplete resection. Hepatogastroenterology 2013;60:46-53.

24. Isomoto H, Ohnita K, Yamaguchi N, et al. Clinical outcomes of endoscopic submucosal dissection in elderly patients with early gastric cancer. Eur J Gastroenterol Hepatol 2010;22:311-317.

25. Chung IK, Lee JH, Lee SH, et al. Therapeutic outcomes in 1000 cases of endoscopic submucosal dissection for early gastric neoplasms: Korean ESD Study Group multicenter study. Gastrointest Endosc 2009;69:12281235.

26. Toyonaga T, Man-i M, East JE, et al. 1,635 Endoscopic submucosal dissection cases in the esophagus, stomach, and colorectum: complication rates and long-term outcomes. Surg Endosc 2013;27:1000-1008.

27. Zullo A, Manta R, De Francesco V, et al. Endoscopic submucosal dissection of gastric neoplastic lesions in Western countries: systematic review and meta-analysis. Eur J Gastroenterol Hepatol 2021;33:e1-e6.

28. Ngamruengphong S, Ferri L, Aihara H, et al. Efficacy of endoscopic submucosal dissection for superficial gastric neoplasia in a large cohort in North America. Clin Gastroenterol Hepatol 2021;19:1611-1619.e1.

29. Hasuike N, Ono H, Boku N, et al. A non-randomized confirmatory trial of an expanded indication for endoscopic submucosal dissection for intestinal-type gastric cancer (cT1a): the Japan Clinical Oncology Group study (JCOG0607). Gastric Cancer 2018;21:114-123.

30. Oda I, Suzuki H, Nonaka S, Yoshinaga S. Complications of gastric endoscopic submucosal dissection. Dig Endosc 2013;25(Suppl 1):71-78.

31. Hanaoka N, Uedo N, Ishihara R, et al. Clinical features and outcomes of delayed perforation after endoscopic submucosal dissection for early gastric cancer. Endoscopy 2010;42:1112-1115.

32. Castillo Contreras O, Maguiña Quispe J, Benites Goñi H, et al. Prevalence of Helicobacter pylori in symptomatic outpatients in red rebagliati (EsSalud) from 2010 to 2013, Lima, Peru. Rev Gastroenterol Peru 2016;36:49-55. 